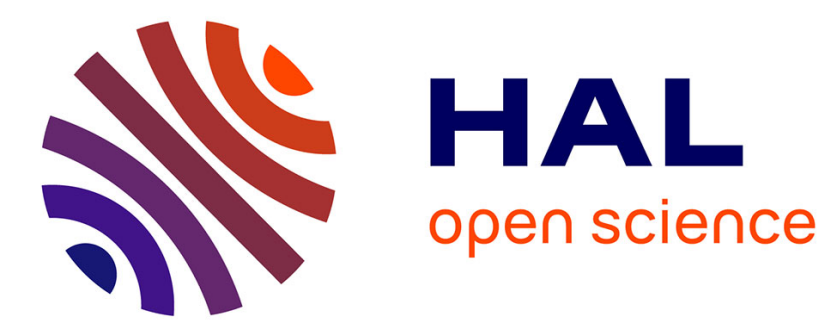

\title{
QUANTUM DESCRIPTIONS OF FREE-ELECTRON LASERS
}

\author{
W. Becker, J. Mciver
}

\section{To cite this version:}

W. Becker, J. Mciver. QUANTUM DESCRIPTIONS OF FREE-ELECTRON LASERS. Journal de Physique Colloques, 1983, 44 (C1), pp.C1-289-C1-311. 10.1051/jphyscol:1983125 . jpa-00222554

\section{HAL Id: jpa-00222554 https://hal.science/jpa-00222554}

Submitted on 1 Jan 1983

HAL is a multi-disciplinary open access archive for the deposit and dissemination of scientific research documents, whether they are published or not. The documents may come from teaching and research institutions in France or abroad, or from public or private research centers.
L'archive ouverte pluridisciplinaire HAL, est destinée au dépôt et à la diffusion de documents scientifiques de niveau recherche, publiés ou non, émanant des établissements d'enseignement et de recherche français ou étrangers, des laboratoires publics ou privés. 


\title{
QUANTUM DESCRIPTIONS OF FREE-ELECTRON LASERS
}

\author{
W. Becker and J.K. McIver \\ Institute for Modem Optics, Department of Physics and Astronomy, University \\ of New Mexico, AIbuquerque, New Mexico 87131, U.S.A.
}

\section{Résumé.-}

Cet article est une revue des différentes descriptions quantiques du laser à électrons libres : (1) Calcul du gain d'après la différence des éléments de matrice pour l'émission et l'absorption d'un photon unique, basé sur les équations de Dirac ou de klein-Gordon ; (2) Description multiphonique basée sur le modèle d'un courant constant interagissant avec un champ électromagnétique quantifié. (3) Résolution de l'équation de Klein-Gordon en présence simultanément du laser et du "wiggler" pris comme champs externes classiques ; ceci donne une description unifiée de tous les régimes à l'exception de l'émission spontanée. (4) Une solution totalement quantifiée de l'Hamiltonien non-relativiste à $N$ corps de Bambini-Renieri defini dans un repère en mouvement. Cette approche illustre bien la différence entre les fluctuations classique et quantique dans le problème du démarrage de l'oscillateur.

\section{Abstract.-}

The various quantum mechanical descriptions of the free-electron laser are reviewed: (I) calculating the gain from the mismatch between the matrix elements for emission and absorption of a single photon, derived from the Dirac- or Klein-Gordon equation; (2) a multiphoton description based on the model of a constant current interacting with a quantized electromagnetic field; (3) solving the Klein-Gordon equation in the presence of both the laser and the wiggler taken as classical external fields, which gives a unified description of all operating regimes but spontaneous emission; (4) a fully quantized small-signal solution of the nonrelativistic many particle Bambini-Renieri Hamiltonian defined in a moving frame. This approach displays the difference between classical and quantum-mechanical fluctuations in the start up.

\section{INTRODUCTION}

The earliest theoretical descriptions of the free electron laser (FEL) were based on quantum mechanics $[1,2]$. In fact, since they involved the quantum-mechanical recoil as the basis of the gain mechanism, it seemed doubtful for a while whether a classical description would be possible, although the independence of the gain of Planck's constant was immediately recognized. Because the early 
quantum-mechanical attempts proposed conflicting results for the gain, there was a wide-spread feeling of relief when it turned out that all relevant features of the FEL could be understood in unambiguous classical terms [3]. Since then the further development of FEL concepts up to the present level of sophistication has employed almost exclusively the classical methods. However, in parallel the quantum-mechanical approach was developed further, as an outcome corroborating beyond any doubts the legitimacy of the former. Hence the question arises as to why still worry about quantum mechanics when it is known to be completely obsolete. The answer consists of three parts. First, quantum mechanics and quantum optics make the concept of a photon available. In general, the determination of the probabilities of photons being emitted or absorbed is much simpler than the evaluation of the corresponding fields. In the opinion of the authors, the simplicity of the quantum-mechanical approach to the basic concepts of the FEL can certainly compete with the simplest classical one-particle approach. In fact, some results though being completely classical were first derived by quantum methods. Second, the important question of the photon statistics is yet unsolved. Attempts have been recently made at determining the photon statistics of a small-gain FEL amplifier; this is, however, completely unrelated to the much more interesting problem of the photon statistics of a FEL oscillator above threshold. The analogous problem for a conventional atomic laser has been exclusively addressed by quantum-mechanical methods [4]. This is not because of logical necessity. Inasmuch as the resulting Glauber function is positive definite, i.e. amenable to a probability interpretation, it could be dealt with by considering classical field ensemble averages as well. However, it appears again to be much easier to count photons. A related question is the start-up problem of the FEL. We shall see that quantities like the power spectrum of spontaneous emission are again classical to an excellent approximation. However, the photon statistics of spontaneous emission exhibit fluctuations which are both of classical and of quantum-mechanical origin: there are classical contributions due to the discreteness of the electron current and quantum-mechanical ones due to the vacuum fluctuations of the electromagnetic field. Whereas the former could be accounted for in a classical approach by noise methods they are already conceptually built into any quantum approach. Third, there are some nonclassical effects in the spontaneous radiation of a dilute electron beam, like photon-antibunching and squeezing, which cannot be obtained by any classical procedure. From a theoretical point of view this puts the FEL beside other systems of quantum optics, like resonance Eluorescence of a two-level atom, which exhibit these phenomena. However, practically it seems to be very doubtful if these effects will ever be accessible to experimental verification.

An outline of this paper is as follows. In section II we use scalar quantum electrodynamics in order to derive the gain from the matrix elements for emission and absorption of a photon by an electron due to the interaction with the wiggler field. This is essentially the line of approach which was followed by the earliest theoretical papers in the field. It immediately yields the connection between gain and spontaneous emission. This is actually valid for a much broader range of phenomena and has already been used for a long time in plasma physics for the determination of plasma instabilities. since the phenomena taking place in a FEL are dominated by multiphoton processes of extremely high order, the accuracy of this approach is really surprising. Hence we turn in section III to a multiphoton analysis based on the model of a constant current interacting with a 
quantized electromagnetic field, which has to be extended in order to include the quantum kinematics of the emission process. It will then be found that taking only the emission and absorption of one photon into account, actually provides correct results for the gain and also largely for the spread even when multiphoton processes completely dominate the real physics. This multiphoton analysis covers the small-signal regime. In order to extend it into the strong signal regime we turn in Section IV to a semiclassical description based on the Klein-Gordon equation in the presence of both the laser and the wiggler field. In the last section we discuss a fully quantized many-electron model. We extract some genuine quantum features of the spontaneous radiation, like photon-antibunching and squeezing, and give a preliminary discussion of the photon statistics of the start-up.

\section{FIRST ORDER CALCULATION OF EMISSION AND ABSORPTION}

The easiest quantum-mechanical and historically the first attempt $[1,2]$ at determining the gain of the FEL proceeded via the evaluation of the matrix elements for the emission and absorption of a photon by an electron due to the presence of the magnetic wiggler field, as represented in Fig. 1. From the point of view of relativistic quantum field theory the FEL constitutes a typical external field problem: the electrons interact with a quantized radiation field on the background of a strong classical external field which cannot be dealt with by perturbation theory. For problems like this the Furry- or bound interaction picture [5] is most convenient. In contrast to the standard interaction picture, in the Furry picture the dynamics of the electron operators takes the external field already into account.

(a)

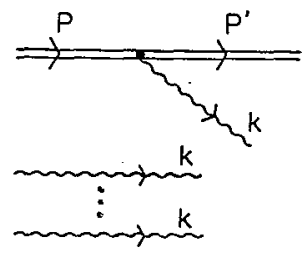

(b)

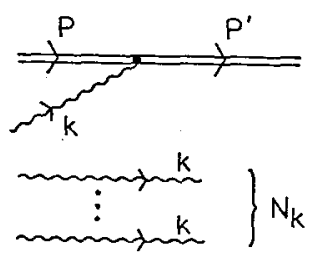

FIGURE 1. Feynman diagrams for the emission (a) and absorption (b) of a photon by an electron. The double lines indicate that the electron wave functios are to be taken in the presence of the wiggler field. The presence of the additional $\mathrm{N}_{\mathrm{k}}$ photons of the same mode affects the transition rate because of Bose statistics.

The matrix element for the emission or absorption of one photon is then to first order given by

$$
M=-i / \hbar<\text { out }\left|\int d^{4} x \mathscr{F}_{I}(x)\right| \text { in }>\text {, }
$$

where the interaction Hamiltonian density

$$
\mathscr{H}_{I}(x)=J_{\mu}(x) A^{\mu}(x)
$$

represents the familiar current-field coupling. 
$A^{\mu}(x)$ is the operator of the quantized laser field in the radiation gauge, and $J_{\mu}(x)$ is the conserved electron current $\left(2 H_{J}(x)=0\right)$ in the presence of the wiggler field. Its explicit form depends upon whether the electron is described by the Dirac- or by the scalar klein-Gordon equation. The appropriate initial and final states are

$$
\begin{aligned}
& |\mathrm{in}\rangle=|\mathrm{p}\rangle\left|\mathrm{N}_{\mathrm{k}}\right\rangle, \\
& \mid \text { out }\rangle=\left|\mathrm{p}^{\prime}\right\rangle\left|\mathrm{N}_{\mathrm{k}} \pm 1\right\rangle,
\end{aligned}
$$

for emission or absorption, respectively. Here p(p') is the initial (final) momentum of the electron and $\mathrm{N}_{\mathrm{k}}$ the number of photons of the considered mode which are initially present. The integration in Eq. (2.1) will be extended over all space and a finite interaction time $T$ or, equivalently, over the wiggler length $L=B_{0} c T$ being unrestricted otherwise. This corresponds to the plane-wave description of Eqs. (2.3) and (2.4).

Since spin is a genuine consequence of relativistic quantum mechanics one expects spin-induced effects to be of relative order

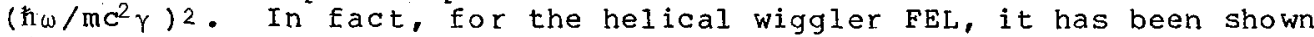
[6] that due to a spin-umklapp process a zeroth harmonic of very low frequency as well as a second harmonic are radiated on axis. Moreover, the fundamental is split depending on the spin state of the electron. These effects are absolutely negligible for the performance of the FEL. Hence describing the electron by the scalar klein-Gordon equation is sufficient*, and the required matrix element of the current is [7]

$$
\begin{aligned}
& \left\langle p^{\prime}\left|J_{\mu}(x)\right| p\right\rangle \\
& =e\left(i \phi_{p}^{*},(x)\left(\vec{a}_{\mu}-\stackrel{\partial}{\partial}_{\mu}\right) \phi_{p}(x)-\frac{2 e}{\hbar} A_{w \mu}(x) \phi_{p}^{*},(x) \phi_{p}(x)\right) .
\end{aligned}
$$

Here $A_{w \mu}(x)$ is the vector potential of the wiggler field, and the wave functions $\phi_{p}(x)$ are solutions of the klein-Gordon equation

$$
\left[\left(\mathrm{i} \partial^{\mu}-\mathrm{eA} \underset{w}{\mu} / \hbar\right)^{2}-\left(\mathrm{mc} \mathrm{c}^{2} / \hbar\right)^{2}\right] \phi_{p}(\mathrm{x})=0 .
$$

On axis the canonical momenta satisfy $\vec{p}_{\vec{A}}=\vec{p} \cdot \vec{A}=0$. Hence the solutions are just exponentials**

$$
\phi_{p}(x)=\left(2 p_{0} v\right)^{-\frac{1}{2}} e^{-\frac{2}{i} p x / \hbar}
$$

* Describing the electron by the Klein-Gordon equation whenever spin effects are not of interest is not admissible in general. It is, however, for the Compton-type processes considered here. ** We use relativistic four-vector notation throughout, such that $\left(a^{\mu}=\left(a^{0}, \vec{a}\right)\right)$

$$
a^{\mu} b_{\mu}=: a b=a^{0} b^{0}-\vec{a} \vec{b}
$$


with $p_{0}=p_{0}=m c^{2} r$ and $v$ the normalization volume. Due to the wiggler field with amplitude $a_{w}$ the electron has acquired an effective mass:

$$
\tilde{\mathrm{p}}^{2}=\mathrm{m}^{2} \mathrm{c}^{4}+\left(e \mathrm{a}_{\mathrm{w}}\right)^{2}=\mathrm{M}^{2} \mathrm{c}^{4}
$$

With all the ingredients specified the evaluation of the matrix elements $M(\underline{t})$ for emission and absorption is rather straightforward: Gain is given by the difference between the rates for emission and absorption, integrated over the phase space of the final electron, divided by the number of initial photons and multiplied by the number $\mathrm{N}$ of electrons. I.e., the gain per pass is

$$
G=\frac{N_{e}}{N_{k}} \int \frac{d^{3} p^{n} \cdot V}{(2 \pi h)^{3}}\left(\left|M^{(+)}\right|^{2}-\left|M^{(-)}\right|^{2}\right) \text {. }
$$

The matrix elements $M^{(+)}$and $M^{(-)}$coincide up to two differences: (1) $M(+)$ includes spontaneous emission. Hence it is proportional to $\mathrm{N}_{\mathrm{k}}+1$, whereas $M^{(-)}$is proportional to $N_{k}$; (2) for fixed electron energy, the line shapes for emission and absorption of a photon are shifted versus each other by a slight recoil contribution. The latter is of purely kinematical origin: for an infinite interaction region energy-momentum conservation requires

$$
\tilde{p}^{\prime}=\tilde{p} \mp \hbar\left(k+k_{0}\right),
$$

where the upper (lower) sign refers to emission (absorption)*. $\tilde{p}=\mathrm{mc}^{2} \gamma$ $\left(1,0,0, \beta_{0}\right), p^{\prime}=m c{ }^{2} \gamma^{\prime}\left(1,0,0, B_{0}^{\prime}\right)$ are the initial and final canonical momenta of the electron, respectively, $\beta_{0}$ and $\beta_{0}$ ' the corresponding longitudinal velocities, $k=(\omega, 0,0, \omega / c)$ is the wave vector of the emitted or absorbed photon and $k_{0}=\left(0,0,0,2 \pi / \lambda_{0}\right)$ that of the wiggler field with period $\lambda_{0}$. For a finite wiggler length, Eq. (2.10) specifies the center of the line shape. In view of Eq. (2.8) it can be solved for the corresponding frequencies

$$
\omega^{( \pm)}=\omega_{0} \beta_{0} /\left(1-\beta_{0} \pm \hbar \omega_{0} / m c^{2} \gamma\right),
$$

where $\omega_{0}=2 \pi c / \lambda_{0}$. Equation (2.11) exhibits the recoil-related frequency shift, which is of central importance for any quantum description of the FEL. Figure 2 shows the well known dependence of gain on frequency which is evident from Eqs. (2.9) and (2.11).

For most imaginable FEL's $\left|M^{( \pm)}\right|^{2}$ in Eq. (2.9) can be expanded to first order around the resonance frequency $\omega_{\text {res }}=\omega_{0} \beta_{0} /\left(1-B_{0}\right)$. This reproduces the well known small-signal cold-beam derivative formula for the gain [3], from which h has cancelled. More general formulae for the gain can be obtained by integrating Eq. (2.9) over an initial electron energy distribution $\mathrm{f}\left(\mathrm{p}_{3}\right)$ with $\int \mathrm{d} \widetilde{\mathrm{p}}_{3} \mathrm{f}\left(\tilde{\mathrm{p}}_{3}\right)=1$ :

\footnotetext{
* The fact that $k_{0}$ enters Eq. (2.10) with the indicated sign is not obvious. To establish it requires a closer look at the matrix element.
} 
FIGURE 2

Line shapes for emission (em) and absorption (abs) of a single photon as a function of frequency for fixed electron energy. Gain at a fixed frequency is proportional to the difference.

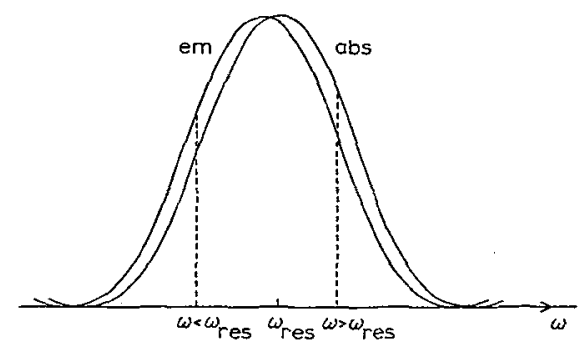

distribution $\mathrm{f}\left(\tilde{\mathrm{p}}_{3}\right)$ with $\int \mathrm{d} \tilde{\mathrm{p}}_{3} \mathrm{f}\left(\tilde{\mathrm{p}}_{3}\right)=1$ :

$$
\begin{aligned}
G_{f} & =\int \tilde{p}_{3} f\left(\tilde{p}_{3}\right) G \\
& =4 \pi\left(e^{2} a_{w}\right)^{2} p_{e} /(\hbar \omega) \int \frac{d \tilde{p}_{3}}{p_{0}^{2}}\left(\frac{\sin x}{x}\right)^{2}\left[f\left(\tilde{p}_{3}+\hbar\left(\omega+\omega_{0}\right) / 2 c\right)\right. \\
& \left.-f\left(\tilde{p}_{3}-\hbar\left(\omega+\omega_{0}\right) / 2 c\right)\right]
\end{aligned}
$$

with

$$
\begin{aligned}
& x=\left[\omega-\left(\omega+\omega_{0}\right) c \tilde{p}_{3} / p_{0}\right] T / 2=\Delta \omega T / 2 \\
& p_{0}=\left[\left(m c^{2}\right)^{2}+\left(e a_{w}\right)^{2}+\left(c p_{3}\right)^{2}\right]^{\frac{1}{2}}
\end{aligned}
$$

and $\mathrm{L}=B_{0} \mathrm{CT}$ the wiggler length and $\rho_{\mathrm{e}}=\mathrm{N}_{\mathrm{e}} / \mathrm{V}$. Again, under almost all conditions, Eq. (2.12) can be expanded so that $\hbar$ cancels and the gain depends on the derivative $\partial f / \partial \tilde{p}_{3}$. Equation (2.12) allows to draw the analogy [8] to population inversion in an ordinary laser.

The rate for spontaneous emission is obtained from Eq. (2.9) by letting $\mathrm{N}_{\mathrm{k}}=0$,

$$
\begin{aligned}
\mathrm{dr}_{\text {spont }} & =\int \frac{\mathrm{d}_{-}^{3} \mathrm{p}^{2} \mathrm{~V}}{(2 \pi \mathrm{h})^{3}}\left|\mathrm{M}^{(+)}\right|{ }^{2} \mathrm{~N}_{\mathrm{k}}=0 \frac{\mathrm{d}^{3} \mathrm{kV}}{(2 \pi)^{3}} \\
& =\frac{\pi\left(\mathrm{e}^{2} \mathrm{a}_{\mathrm{w}}\right)^{2} \mathrm{~T}^{2}}{\hbar \omega(\mathrm{mc} \gamma)^{2} V}\left(\frac{\operatorname{sinx}}{\mathrm{x}}\right)^{2} \frac{\mathrm{Vd}^{3} \mathrm{k}}{(2 \pi)^{3}},
\end{aligned}
$$

hence we can rewrite Eq. (2.12) as 


$$
\begin{aligned}
G_{f} & =\left.(2 \pi)^{3} \rho_{e} \int d \tilde{p}_{3} \frac{d \Gamma \text { spont }}{d^{3} k}\right|_{\theta=0}\left[f\left(\tilde{p}_{3}+\hbar\left(\omega+\omega_{0}\right) / 2 c\right]\right. \\
& \left.-f\left(\tilde{p}_{3}-\hbar\left(\omega+\omega_{0}\right) / 2 c\right)\right] .
\end{aligned}
$$

Here the spontaneous emission rate should be taken in the forward direction, as indicated by $\theta=0$. Equation (2.15) specifies the gain in terms of spontaneous emission. It is valid for a much wider class of phenomena than derived here. Only the kinematics of the emission process which enters the argument of the function $f$, must be adapted to the particular situation. The importance of Eq. (2.15) lies in the fact that the spontaneous transition rate for free-free-transitions which we are considering here (which might be referred to as the rate for classical bremsstrahlung as well) can to an excellent approximation be derived from classical electrodynamics, once the electron trajectories in the presence of merely the external (wiggler-) field (not the laser field) have been specified. This can in many cases be done exactly, or at least in some analytical approximation.

The approach outlined above is one of the easiest methods to evaluate the gain for the FEL and a variety of similar devices. Its simplicity is, however, somewhat deceptive. We only considered the emission of a single photon, although it is well known that, e.g. under the conditions of the stanford FEL, one electron can emit or absorb about ten thousand photons during one pass through the wiggler. The deficiency of our present approach becomes apparent if one asks for quantities other than the gain, like spread or bunching. The origin of the difficulty lies in the fact that we interpreted the matrix elements $M( \pm)$ in terms of transition probabilities, which is, of course, their correct meaning. Ths implies, however, that they have to be smaller than unity. If we had plugged in numbers at an early stage we would have found that for reasonable intensities of the stimulating field the alleged transition probabilities are much larger than one. The failure is, of course, due to our restriction to first order processes. In the framework of the model of a constant current interacting with a quantized radiation field it has been shown long ago [9] that if proper account is taken of all multiphoton processes, the meaning of the first order quantity $|\mathrm{M}(+)|^{2} \mathrm{~N}_{\mathrm{k}}=0$ changes. It does no longer give the probability of one photon emission, but specifies instead the mean number of emitted photons. This makes plausible the fact that we obtained the correct small signal gain by merely considering first order processes. It also reveals the limits of the present approach: it fails in the strong-signal regime when the state of the electron is significantly affected by the interaction with the laser field. Yet, as long as one has these restrictions in mind, this method can be and has been used to provide a quick and easy access to the small signal gain for various situations. In addition to the standard EEL $[1,6,10]$ it has been used for stimulated Cerenkov radiation [11], the Compton laser $[2,12]$, and under the heading of the "Einstein coefficient method" for the FEL with an axial magnetic guide field [13] as well as, in a different field, for the calculation of plasma instabilities [14].

We shall give a more accurate description in the following sections: in the next section we shall have a closer look at the multiphoton processes using the exactly solvable constant current 
model as the zeroth order approximation. Thereafter we shall return to the Klein-Gordon equation incorporating now both the wiggler and the laser as external fields. The latter approach will turn out to be applicable also to the strong signal regime.

\section{MULTIPHOTON PROCESSES}

The fact that the electrons leave the wiggler with a significant energy spread corresponding to an energy exchange with the laser field of $\sim 10^{5}$ photons in either direction, makes evident the fact that the phenomena in the FEL are governed by multiphoton processes of extremely high order. On the other hand the absolute change in the electron energy is still quite small. Hence, it is suggestive to use the model of a prescribed classical current interacting with a quantized radiation field as a zeroth approximation to the FEL. This has the advantage of being readily exactly solvable. It is clear, though, that this has to be improved upon, since Eq. (2.15) shows that gain is lost if the backaction of emission and absorption on the electron is neglected. Since this is basically specified by energy-momentum conservation as exhibited in Eq. (2.10) it will turn out that it can easily be incorporated into the zeroth order approximation. Since this approach has been worked out rather extensively [15-17] we shall skip many of the details in this section.

An exact formal solution of the fully quantized problem is given by the time-evolution operator

$$
S(T)=I \exp (-i / h) \int_{-T / 2}^{T / 2} d t \int d^{3} x J C_{I}(x),
$$

where $I$ is the time-ordering operator. S(T) transforms an initial state at $t=-T / 2$, as e.g. given by Eq. (2.3), into a final state at $t=T / 2$ after the interaction mediated by the wiggler field. The first order expansion of $\mathrm{Eq}$. (3.1) has been investigated in the last section. We will restrict ourselves to radiation on axis and one mode of the laser field. Hence, $S(T)$ acts on the initial state (2.3) like

$$
S(T)|N, p\rangle=\sum_{n=-N}^{\infty}\left|N+n, p-n \hbar\left(k+k_{0}\right)\right\rangle\langle N+n|S(T)| N\rangle \text {. }
$$

Here we dropped the subscript $k$ on $N$ and omitted indicating the electron states on the matrix element due to their one-to-one relationship to the change in photon number.

We obtain the above mentioned model if we replace the current operator $J_{\mu}(x)$ in Eq. (3.1) by the classical current

$$
\vec{J}^{\text {class }}(x)=\operatorname{ec} \vec{\beta}(\vec{x}) \delta(\vec{x}-\vec{x}(t))
$$

of the electron in the wiggler ficld which is obtained by solving the Lorentz force equation. The transition probabilities 


$$
w_{n}^{(N)}(z)=|<N+n| S(T)|N>|^{2}
$$

from an initial state with $\mathrm{N}$ photons to a Einal state with $N+n$ photons can then readily be evaluated. They depend on the quantity

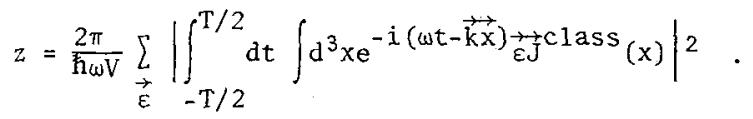

Here the sum is over two mutually orthogonal polarization vectors $\vec{\varepsilon}$ with $\overrightarrow{k \varepsilon}=0$. For the helical wiggler FEL we have

$$
z=\frac{\pi e^{4} a_{w}^{2} T^{2}}{h \omega V(m \gamma c)^{2}}\left(\frac{\sin x}{x}\right)^{2}
$$

where the parameter $x$ is related to the detuning and was defined in Eq. (2.13). Comparison with Eq. (2.14) shows

$$
z=\frac{(2 \pi)^{3}}{V} \frac{d \Gamma \text { spont }}{d^{3} k} \int \frac{d^{3} p^{2} \cdot V}{(2 \pi \hbar)^{3}}\left|M^{(+)}\right|^{2} N_{k}=0
$$

We only mention the limiting expression for $w_{n}(N)(z)$ for $N>1$, $z \ll 1$ (cp. also [181)

$$
w_{n}^{(N)}(z)=J_{n}(2 \sqrt{N z})^{2}\left(1+0\left(n^{2} / N\right)\right)
$$

which allows for photon transfers up to

$$
\mathrm{n}_{\max }=2 \sqrt{\mathrm{Nz}}
$$

The symmetry of Eq. (3.8) upon $n \rightarrow-n$ shows that there is no gain for $\mathrm{N} \gg 1$, pointing to the necessity of improving the model.

It is apparent from Eq. (3.6) that $z$ depends on the detuning $\omega-\omega_{\text {res }}=\omega-\omega_{0} \beta_{0} /\left(1-\beta_{0}\right)=\Delta \omega /\left(1-\beta_{0}\right)$.

Actually, the probability $w_{n}^{(N)}$ (z) for the emission of n photons 

should not be centered at $\omega={ }^{\omega}$ res, but at the recoil-shifted
frequency

$$
\omega^{(n)}=\omega_{0} \beta_{0} /\left(1-\beta_{0}+n \hbar \omega / m c^{2} \gamma\right),
$$

Which is obtained from energy-momentum conservation, i.e. $\tilde{\mathrm{p}}^{\prime}=\hat{\mathrm{p}}-\mathrm{nh}\left(\mathrm{k}+\mathrm{k}_{0}\right)$, in the same manner as Eq. (2.11). Hence we take the all important recoil into account by replacing by hand

$$
w_{n}^{(N)}(z) \rightarrow w_{n}^{(N)}\left(z_{n}\right),
$$

where $z_{n}$ is obtained from $z$ by replacing

$$
\Delta \omega \rightarrow\left(1-\beta_{0}\right)\left(\omega-\omega^{(n)}\right) .
$$

For the small signal regime, a first order expansion

$$
w_{n}^{(N)}\left(z_{n}\right)=w_{n}{ }^{(N)}(z)+n \Delta z \frac{\partial w_{n}^{(N)}(z)}{\partial z}+\ldots
$$

turns out to be sufficient, which defines the quantity

$$
\Delta z=\left(z_{n}-z\right) / n \text {. }
$$

For the helical wiggler FEL,

$$
\Delta z=\frac{\pi e^{4} a^{2} \omega_{0} T^{3}}{2 V(m \gamma c)^{3} c} \frac{\partial}{\partial x}\left(\frac{\sin x}{x}\right)^{2} .
$$

If necessary, the expansion (3.14) can be carried further [19], yielding saturation corrections to the small-signal gain.

The gain is then proportional to

$$
<n>=\sum_{n=-N}^{\infty} n w_{n}^{(N)}\left(z_{n}\right) \cong z+\Delta z(2 N+\cdot 1+2 z),
$$

where the first term specifies spontaneous and the second term stimulated emission. Since $\Delta z \ll z \ll l$ the last two terms are insignificant. In view of Eq. (3.17) and (3.7) we conclude that $|M( \pm)|^{2} N_{k}=0$ actually specifies the number of emitted photons and 
not, as concluded from the first order calculation, the transition probability for the emission of one photon [9]. It is instructive to consider the limit $\mathrm{Nz} \ll I$ which corresponds to stimulating field intensities so small that only one photon processes occur [20]. In this limit only

$$
\begin{aligned}
& w_{1}^{(N)}(z)=(N+1) z, \\
& w_{0}^{(N)}(z)=1-(2 N+1) z, \\
& w_{-1}^{(N)}(z)=N z
\end{aligned}
$$

survive. Calculating the gain from Eq. (3.18) we obtain

$$
<n>=w_{1}^{(N)}\left(z_{1}\right)-w_{1}^{(N)}\left(z_{-1}\right)=z+\Delta z(2 N+1)
$$

which agrees with Eq. (3.17) apart from the completely insignificant

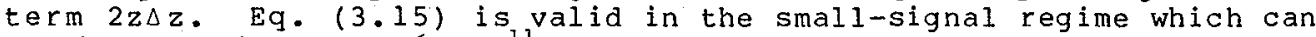
be shown to imply Nz ${ }^{2} 10^{11}$. Hence in view of Eq. (3.9) we summed over $z 10^{5}$ terms and yet the same result can be obtained as trivially as from Eq. (3.19)! This explains the success of the first order approach of section 2. The deeper physical reason for this amazing fact has to be sought in the infrared regime of guantum electrodynamics.

In analogy to the gain the spread can be evaluated, and the gain-spread relation [21] is obtained immediately. In view of Eq. (3.2) bunching of the electrons after leaving the wiggler can be inferred from the matrix elements $\langle\mathrm{N}+\mathrm{n}|\mathrm{S}(\mathrm{T})| \mathrm{N}\rangle$. Bunching turns out to be related to gain, and not to the spread. Everything is expressible in terms of the two quantities $z$ and $\Delta z$.

The limits of applicability of the above approach are defined by the condition that the recoil as exhibited in Eq. (3.11) be small up to the maximum value of $n$ given in Eq. (3.9). It would be unnecessarily stringent to demand that it be small compared to $\Delta \omega$. It should be small with respect to the width $T^{-1}$ of the line shape function $z(\Delta \omega)$. This yields the condition

$$
\frac{1}{2} \mu^{2}:=\frac{\mathrm{e}^{2} \mathrm{~B}_{0} \mathrm{E}_{\mathrm{L}} \mathrm{T}^{2}}{(\mathrm{~m} \gamma \mathrm{c})^{2}} \ll 1,
$$

where $B_{0}$ is the magnetic field strength of the wiggler and $E_{L}$ the electric field strength of the laser. This is the usual condition for the small signal regime [22]. Some flaw of the approach must be mentioned, too. After the by hand-introduction of the recoil the "probabilities" $w_{n}(N)(z)$ are no longer normalized to unity; in fact, one can easily compute

$$
\sum_{n=-N}^{\infty} w_{n}^{(N)}\left(z_{n}\right)=1+\Delta z
$$


The possible impact of this deficiency can be estimated by renormalizing the transition probabilities to unity, defining

$$
\tilde{w}_{n}{ }^{(N)}\left(z_{n}\right)=w_{n}{ }^{(N)}\left(z_{n}\right) /(1+\Delta z) .
$$

obviously, this does not alter the dominant terms in the gain or spread.

The main advantage of the approach outlined in this section seems to be its conceptual simplicity and easy applicability to various free-electron devices. Only the two quantities $z$ and $\Delta z$ have to be specified, where the first is related to spontaneous emission and the latter incorporates the quantum kinematics. Several examples other than the helical wiggler FEL have been sketched in Ref. 17, among these the Cerenkov laser more extensively in Ref. 23. Essentially along the same lines a refractive index has been added to the standard FEL [24]. However, it seems not to be feasible to extend this method to the strong-signal regime. Moreover, genuine quantum features derived from it, which are bound to be very small in any event, would not be reliable due to the above mentioned built-in lack of complete consistency. Hence, we shall delay the discussion of photon statistics of the small-gain FEL-amplifier to section 5, where a rigorous fully quantized solution will be presented.

\section{STRONG SIGNAL GAIN}

Although the small signal gain is easily derived both from a classical and quantum-mechanical point of view, the calculation of the strong signal or saturated gain is a more formidable task. The only nonclassical derivations of the strong signal gain treat the electron quantum-mechanically and the wiggler and electromagnetic fields classically [25-28]. Because spin-effects have been shown to be unimportant [6], the Klein-Gordon equation with classical wiggler and electromagnetic fields is used to describe the electron

$$
\left[\frac{\partial^{2}}{\partial z^{2}}-\frac{1}{c^{2}} \frac{\partial^{2}}{\partial t^{2}}-\frac{M^{2} c^{2}}{\hbar^{2}}-\frac{2 e^{2}}{\hbar^{2} c^{2}} \vec{A}_{W} \cdot \vec{A}_{L}\right] \psi=0,
$$

where

$$
\begin{aligned}
& \vec{A}_{W} \cdot \vec{A}_{L}=\frac{a_{W^{\prime}} a_{L}}{2}\left[e^{i\left\{\left(k+k_{0}\right) z-\omega t\right\}}+c \cdot c \cdot\right], \\
& M^{2}=m^{2}+\frac{e^{2}}{c^{4}}\left(a_{W}^{2}+a_{L}^{2}\right),
\end{aligned}
$$

and $\mathrm{m}$ is the rest mass of the electron.

The solution of Eq. (4.1) has been treated along two lines. In one case [27], a coordinate transformation is used to transform the Klein-Gordon equation to a Mathieu equation which is then solved exactly using a Green's function in order to incorporate appropriate initial conditions. Via asymptotic expansions for the Mathieu functions the well known expression for the small signal gain is derived as is a simple analytic expression for the strong signal gain. 
The second method $[25,26,28]$ is mathematically more complex but has the advantage of providing a clear physical picture. It is this method that is discussed below.

The wave function that satisfies Eq. (4.1) may be written as superposition of $\mathrm{plane}$ waves in the form

$$
\psi=\sum_{n=-\infty}^{\infty} c_{n} \exp \left\{\frac{i}{\hbar}\left(p_{n} z-\varepsilon_{n} t\right)\right\},
$$

where

$$
\begin{aligned}
& p_{n}=p+n \hbar\left(\omega+\omega_{0}\right) / c \\
& \varepsilon_{n}=c \sqrt{p_{n}^{2}+M^{2} c^{2}} \sim \varepsilon+\frac{n h c\left(\omega+\omega_{0}\right) p}{\varepsilon}+\frac{n^{2} \hbar^{2} c^{2} m^{2} \omega^{2}}{\varepsilon^{3}}
\end{aligned}
$$

and $\varepsilon=\varepsilon_{0}$ and $p$ are the energy and momentum of the electron before the interaction is switched on at $t=0$. With this wave function the klein-Gordon equation reduces to a second-order differential-difference equation. After several approximations followed by another transformation, $c_{n}(t)=a_{n}(t) \exp \left\{i\left(\varepsilon_{n}-n \omega-\varepsilon\right) t\right\}$, the following equation is derived $[25,28]$ :

$$
i \hbar \frac{\partial a}{\partial t}-\varepsilon_{\text {anh }}\left(2 n \frac{\Delta}{\hbar \omega}+n^{2}\right) a_{n}=\varepsilon_{i n t}\left(a_{n+1}+a_{n-1}\right)
$$

with $a_{n}(t=0)=\delta_{n_{0}}$, where

$$
\varepsilon_{\text {anh }}=\frac{\left(\mathrm{mc}^{2} \hbar \omega\right)^{2}}{\varepsilon^{3}}
$$

is the anharmonic energy,

$$
\varepsilon_{\text {int }}=\frac{e^{2} a_{W} a_{L}}{2 \varepsilon}
$$

is the interaction energy and

$$
\Delta=\varepsilon-M c^{2} \sqrt{\omega / 2 \omega_{0}} \simeq-\frac{m c^{2} \gamma}{2 \omega_{0}} \Delta \omega
$$

is the detuning from resonance. The quantities $t, \varepsilon_{\text {anh }}$ and $\varepsilon_{\text {int }}$ may be combined to form nondimensional parameters

$$
\begin{gathered}
n=t \varepsilon_{\text {int }} / \hbar, \quad \rho=\varepsilon_{\text {int }} / \varepsilon_{\text {anh }}, \\
\beta=t \varepsilon_{\text {anh }} / \hbar, \quad \mu=2 n / \sqrt{\rho}=2 t \sqrt{\varepsilon_{\text {int }} \varepsilon_{\text {anh }}} / \hbar .
\end{gathered}
$$

These nondimensional quantities can be interpreted in terms of physical concepts: $\eta$ and $\sqrt{\rho}$ determine the number of photons emitted or absorbed by an electron for the case of small-signal and strong-signal conditions respectively, $B$ determines the small quantum correction to the classical expression for the gain and $\mu$ is the previously introduced saturation parameter. 
Once the coefficients $a_{n}(t)$ are known the energy $\Delta \varepsilon$ emitted by an electron in a time $t$ is calculated using

$$
\Delta \varepsilon \simeq-\hbar \omega \sum_{n} n\left|a_{n}(t)\right|^{2} .
$$

The gain G for a beam of electrons with number density $\rho$ is related to the emitted energy by

$$
G=\frac{4 \pi \rho e^{c^{2}}}{\omega^{2} a_{L}^{2}} \Delta \varepsilon
$$

Although the differential-difference equation (4.5) is more convenient to use in order to understand the physics of the interaction, it is difficult to deal with analytically. For this reason, it is convenient to transform Eq. (4.5) back to a differential equation by defining the function

$$
\phi(x, t)=e^{i \Delta t / \hbar \omega} \sum_{n} a_{n}(t) e^{i n x}
$$

in the region $0 \leqslant x<2 \pi$. This function then satisfies the equation

$$
i \frac{\partial \phi}{\partial t}=-\varepsilon_{\text {anh }}\left(\frac{\partial^{2}}{\partial x^{2}}-2 \rho \cos x+\frac{\Delta^{2}}{(\hbar \omega)^{2}}\right) \dot{\phi}
$$

with the initial condition

$$
\phi(x, t=0)=e^{i \Delta x / \hbar \omega}
$$

The method of solution of Eqs. (4.5) and (4.13) depends upon the size of the saturation parameter $\mu$. If $\mu<1$ then the number of excited levels in the potential is approximately given by $\eta[25]$. In this case, the anharmonicity represented by the term $n^{2} \varepsilon_{\text {anh }}$ in Eq. (4.5) is relatively unimportant and can be treated as a perturbation. This term is the origin of the second derivative in Eq. (4.43). So for $\mu<1$, the term $\left(\partial^{2} \phi / \partial x^{2}\right)$ is treated as a perturbation. Therefore

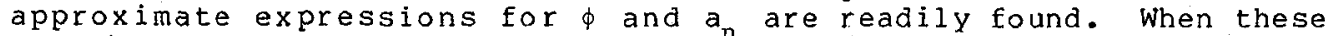
solutions are used to calculate the gain via Eqs. (4.10) and (4.11) the resulting expression is the well known formula for the small signal gain $[25,28]$.

When $\mu>1$ this technique no longer applies since the number of excited levels is now given by $\sqrt{\rho}$ which is a 1 arge number ( $10^{5}$ for the stanford case [30]) so that the anharmonicity is an important factor [25]. On the other hand, the depth of the effective potential $2 \rho$ cosx in Eq. (4.13) is very large. For this reason the potential can be expanded about its minimum $x=\pi$ in a power series

$$
2 \rho \cos x \simeq 2 \rho\left(1-\frac{(x-\pi)^{2}}{2}+\frac{(x-\pi)^{4}}{24}\right)
$$

for a large number of low lying states. In particular when the number of excited states is given by $\sqrt{\rho}$, this expansion is valid.

A substitution of expansion (4.15) into Eq. (4.13) reduces the latter to the Schrodinger equation for an anharmonic oscillator. 
Approximate solutions for this equation are also readily available. The expression for the energy emitted by an electron is then found to be $[25]$

$$
\Delta \varepsilon=\Delta\left\{1-\sqrt{\frac{2}{\mu \pi}}\left[\cos \left(\mu\left(1-\frac{\Delta^{2}}{16 \Delta_{m}^{2}}\right)\right)+\sin \left(\mu\left(1-\frac{\Delta^{2}}{16 \Delta_{m}^{2}}\right)\right)\right]\right\},
$$

where $\Delta_{0}=\hbar \omega \sqrt{\rho}$. In order to derive this expression, it was necessary to impose $\frac{m}{2}$ restriction on the size of detuning [25]. The conditions for the applicability of Eq. (4.16) are now given by $\mu>1,|\Delta|<\Delta_{m}$.

Equation (4.16) along with the corresponding expression for the small signal regime gives an almost complete picture of the emitted energy as a function of $\mu$. This dependence is shown in Fig. 3 . The only missing information is the behavior in the region $\mu \simeq 1$ which can be predicted from Fig. 3 and the behavior when $|\Delta|>\Delta_{\mathrm{m}}$ and $\mu>1$.

\section{FIGURE 3}

Normalized plot of $\Delta \varepsilon(\mu)$. The dashed portion of the curve represents the region intermediate between the small-signal regime $(\mu \ll l)$ and the strong signal regime $(\mu>>1)$.

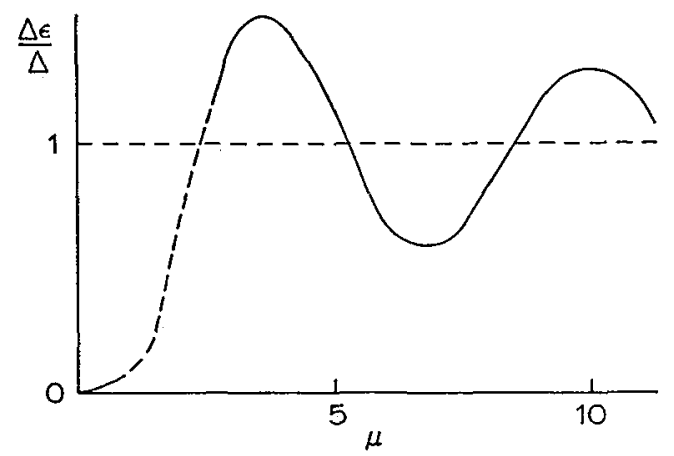

In order to investigate the latter problem it is necessary to again turn our attention to $\mathrm{Eq}$. (4.5) and determine the relative sizes of the terms. When $|\Delta| \simeq \Delta$ the detuning, the anharmonicity and the interaction terms are of the same order [25]. If on the other hand $|\Delta|>\Delta_{m}$ then the anharmonicity is relatively small and can be treated as a perturbation. This is just the approximation that was used for the small signal gain. Therefore, in the region $\mu>1$ and $|\Delta|>\Delta \mathrm{m}$, the system is again in the region of small signal gain.

If the energy emitted by an electron or the gain is considered as a function of the detuning, then in the region where $\mu>1, \Delta_{m}$ determines the boundary between the region in which Eq. (4.16) is valid $\left(|\Delta|<\Delta_{m}\right)$ and Eq. (2.12) is valid $\left(|\Delta|>\Delta_{m}\right)$. Consequently, the quantities $\Delta \varepsilon$ and $G$ increase with increasing $|\Delta|$ when $\Delta<\left|\Delta_{m}\right|$ and decrease with increasing $|\Delta|$ when $|\Delta|>\Delta_{m}$. Therefore in the saturation region $\Delta \varepsilon$ as a function of $\Delta$ should be a maximum when $\Delta \simeq \Delta_{m}$. Equation (4.16) and (4.11) provides simple expressions for the maximum radiated energy $\Delta \varepsilon_{\mathrm{m}}$ and for the maximum $\mathrm{G}_{\mathrm{m}}$ when $\mu>1$

$$
\begin{aligned}
& \Delta \varepsilon_{\mathrm{m}} \simeq \Delta_{\mathrm{m}} \\
& \mathrm{G}_{\mathrm{m}} \simeq \frac{4 \pi \rho_{\mathrm{e}} \mathrm{\Delta}_{\mathrm{m}}}{\mathrm{E}_{0}{ }^{2}}=\frac{2 \sqrt{2} \rho_{\mathrm{e}} \mathrm{e}^{\sqrt{\mathrm{B}_{0}}}}{\omega_{0} \mathrm{c} \mathrm{E}_{0}^{3 / 2}}
\end{aligned}
$$

The wavelength $\lambda_{\mathrm{m}}$ corresponding to $\Delta=\Delta_{\mathrm{m}}$ shifts with increasing laser field strength towards larger values according to 


$$
\lambda_{\mathrm{m}}-\lambda_{\text {res }}=\frac{2 \Delta_{\mathrm{m}}}{\varepsilon} \lambda_{\text {res }}
$$

where $\lambda_{\text {res }}=\lambda_{0} \varepsilon^{2} / 2 \mathrm{M}^{2} \mathrm{c}^{4}$ is the small signal resonance wavelength. If the location of the horizontal axis in Fig. 12 of Ref. [29] is assumed to correspond to the wavelength $\lambda_{s s}$ at the maximum of the small-signal gain, then it is necessary to calculate the wavelength shift $\lambda_{s s}$ - $\lambda_{\text {res }}$ in order to make a comparison with this experiment. This shift is given by

$$
\lambda_{\text {ss }}-\lambda_{\text {res }}=\frac{2.6 \lambda_{\text {res }}^{2}}{\pi \mathrm{L}}\left(\frac{\varepsilon}{M c^{2}}\right)^{2}
$$

so that

$$
\lambda_{\mathrm{m}}-\lambda_{\mathrm{ss}} \simeq 8 \times 10^{-3} \mu \mathrm{m}
$$

where use has been made of the numbers in Ref [29]. This prediction not only agrees with the direction of the observed wavelength shift but is also within a factor of two of the magnitudes.

Although the derivation of the strong-signal gain was carried out using quantum-mechanical methods, the resulting expression is classical. In fact, Eq. (4.16) has been derived from classical considerations [31]. Comparison of the results of this section can also be made with the results of the numerical solution of the classical equations in Ref. [32]. Here qualitative agreement is achieved. Any closer comparison is difficult because the numerical calculations did not investigate the dependence of the physical quantities on all the available parameters.

The model that has been used in this section is completely general as far as stimulated emission is concerned. Furthermore, an exact solution for the Klein-Gordon Eq. (4.1) can be written down. Because this solution is written in terms of Mathieu functions it has not yet proved to be very useful. With some effort this approach can also be extended to include other stimulated free-electron processes like Cerenkov radiation [23]. The obvious shortcoming of this model is the neglect of spontaneous emission which cannot easily be incorporated.

\section{FULLY QUANTIZED MANY-ELECTRON MODEL FOR SPONTANEOUS EMISSION AND THE SMALL-SIGNAL REGIME}

In this section we shall reevaluate the time-evolution operator (3.1). Instead of starting from a prescribed classical current and reintroducing the recoil by hand as we did in Section 3, we shall now attempt a rigorous first order expansion with respect to the recoil. We shall also treat the general case of an arbitrary though fixed number $\mathrm{N}_{e}$ of electrons since this will turn out to be of crucial importance for the start-up problem. Most of the material of this section is for $\mathrm{N}_{\mathrm{e}}=1$ contained in Ref. 32, for arbitrary $\mathrm{N}_{e}$ in [33].

We will in the following restrict ourselves to the helical wiggler FEL. In this case it is advantageous to transform to a moving frame of reference, the so called Bambini-Renieri frame [34]. This can be defined by the condition that the explicit time-dependence of the classical Hamiltonian be eliminated. The longitudinal electron motion is then nonrelativistic, resonance i.e. zero small-signal gain 
occurring if the electron is at rest. The quantized many-particle Hamiltonian which we shall employ [33] is then

$$
\begin{aligned}
& \mathrm{H}=\mathrm{H}_{0}+\mathrm{H}_{\mathrm{I}}, \\
& \mathrm{H}_{\mathrm{O}}=\sum_{i=1}^{\mathrm{N}_{\mathrm{e}}} \frac{\mathrm{p}_{i}^{2}}{2 \mathrm{M}}+\mathrm{h}_{\omega \mathrm{a}^{+} \mathrm{a},} \\
& \left.\mathrm{H}_{\mathrm{I}}=\mathrm{ihg} \sum_{i=1}^{\mathrm{N}_{\mathrm{e}}}\left(\mathrm{a}^{+} \mathrm{e}^{-i(\mathrm{kz}} \mathrm{i}+\omega \mathrm{t}\right)-\text { h.c. }\right) .
\end{aligned}
$$

Here $\mathrm{N}_{e}$ is the total number of electrons, $p_{i}$ and $z_{i}\left(i=1, \ldots N_{e}\right)$ are the operators of momentum and position of the i-th electron, [ $\left.z_{i}, p_{j}\right]$ $=i \hbar \delta . M$ is the effective electron mass defined in Eq. $+(2.8)$ which takes the transverse motion into account. a and a are the annihilation and creation operators of the considered laser mode, $\left[a, a^{\dagger}\right]=1$, and $w \simeq \mathrm{ck} / 2$. All quantities in Eqs. $(5,1-3)$ refer to the moving frame. The coupling constant is

$$
g=\frac{e^{2} a}{M c}\left(\frac{2 \pi}{\hbar \omega}\right){ }^{\frac{1}{2}}
$$

The Hamiltonian (5.1-3) deviates from the Bambini-Renieri Hamiltonian commonly used in that it does not contain a quantized wiggler field. The common procedure $[34,20]$ is to first transform to an extremely relativistic frame so that the wiggler field can be replaced by a radiation field (this is the Weizsäcker-Williams approximation (WWA)), which is then quantized, and then to transform to a frame where the laser and the wiggler photons have the same frequency. This allows for an appealing interpretation of the gain mechanism in the FEL as a conversion of "wiggler photons" into laser photons $[20,35]$. One has to bear in mind, however, that the wiggler. field is a paradigm of a classical external field so that its quantization via the WWA cannot have any physical significance. The Hamiltonian (5.1-3) does not involve the WWA, the only inherent approximation is a nonrelativistic expansion. It agrees, however, with the standard BR-Hamiltonian (if in the latter the quantized wiggler field is taken as classical) apart from minor differences in the relation of the respective parametes to those in the lab frame. It should be noted that a simple form like (5.1-3) is only obtained for the helical wiggler. In general the transverse electron motion is nontrivial and there is no frame in which the electron is completely nonrelativistic.

As in Eq. (3.1) we transform to the interaction picture *

$$
\begin{aligned}
& H_{I}{ }^{(t)}=e^{i H_{0} t / h_{H}} e^{-i H_{0} t / \hbar} \\
& =i \hbar g \sum_{i=1}^{N_{e}}\left(\exp \left[-i t\left(\hbar k^{2}+2 k p_{i}\right) / 2 M\right] A_{i}^{\dagger}-\text { h.c. }\right) \text {. }
\end{aligned}
$$

\footnotetext{
* In the present case, once $M$ is identified with the effective mass, the interaction picture and the Furry picture agree. Again, this is only true for the helical wiggler.
} 
Here we introduced the operators $[36,37]$

$$
A_{i}=a e^{i k z} \dot{i}
$$

with $A_{i}^{\dagger} A_{i}=a^{\dagger} a,\left[A_{i}, A_{j}\right]=0$, but

$$
\left[A_{i}, A_{j}^{\dagger}\right]=e^{i k\left(z_{i}-z_{j}\right)}
$$

The fact that the last commutator is for $\mathrm{N}_{\mathrm{e}}>1$ still an operator, makes the many-particle case considerably more involved than the one-particle case. Expressed differently, for many electrons the set of accessible final states from a specified initial state is no longer one-dimensional, as it was in Eq. (3.2). The time-evoution operator is then

$$
\mathrm{S}(\mathrm{T})=I \exp \left(-\mathrm{i} / \mathrm{h} \int_{-\mathrm{T} / 2}^{\mathrm{T} / 2} \mathrm{dt} \mathrm{H}_{\mathrm{I}}(\mathrm{t})\right) .
$$

If it were not for the operator $p_{i}$ in the exponential of the last line of Eq. (5.5) the evaluation of Eq. (5.8) would be trivial and we would essentially be back to the classical current results of section 3 , having again lost gain on the way. To do better we let

$$
p_{i}=p_{0}+\lambda\left(p_{i}-p_{0}\right)\left(i=1, \ldots N_{e}\right)
$$

where $p_{0}$ is a common c-number to be identified later with the initial electron momentum. We then expand to first order in $\lambda$ letting $\lambda=1$ in the end. The zeroth order term with $p_{i}=p_{0}$, for $\mathrm{N}=1$, reproduces the zeroth order results of section 3 . The first order term introduces the modifications related to gain in a rigorous way. We take as the initial state, generalizing Eq. (2.3)

$$
\mid \text { in }>=|\bar{p}(1)>\ldots| \vec{p}\left(N_{e}\right)>\mid N>
$$

i.e. $N_{e}$ electrons in identical momentum eigenstates and a photon number state. For the current densities of interest we can safely ignore Fermi statistics. Generalizations of the state (5.10) are discussed in Ref. 33. They do not seem to change the results significantly.

We obtain for gain and spread, respectively,

$$
\begin{aligned}
& \Delta N=\left\langle i n\left|S(T){ }^{\dagger}{ }^{\dagger} a s(T)\right| i n\right\rangle-N=N_{e} j^{2}-\frac{h k^{2}}{M}(2 N+1) N_{e} j j^{\prime} \\
& -2 \frac{g^{2} \hbar k^{2}}{\beta^{2} M} N_{e}\left(N_{e}-1\right) j\left(j^{\prime}-\beta j^{\prime \prime}\right)+N_{e} \delta, \\
& \Delta\left(N^{2}\right)=\left\langle i n\left|S(T)^{\dagger}\left(a^{\dagger} a\right)^{2} S(t)\right| i n>-<i n\left|S(T)^{\dagger} a^{\dagger} a_{S}(T)\right| i n\right\rangle^{2} \\
& =N_{e}\left(N_{e}-1\right) j^{4}+(2 N+1) N_{e}\left[j^{2}-2 \frac{\hbar k^{2}}{M} N_{e} j^{3} j \prime\right.
\end{aligned}
$$




$$
\begin{aligned}
& \left.-\frac{2 g^{2} \hbar k^{2}}{\beta^{2} M}\left(N_{e^{-1}}\right) j\left(j^{\prime}-\beta j^{\prime \prime}\right)+\delta\right] \\
& -\frac{h k^{2}}{M} N_{e}\left[j j^{\prime}+4 g^{2} \beta^{-2}\left(N_{e}-1\right)\left(N_{e}-2\right) j^{3}\left(j^{\prime}-\beta j^{\prime \prime}\right)\right] \\
& +4 N_{e}\left(N_{e}-1\right) j^{2} \delta .
\end{aligned}
$$

Here we used the abbreviations

$$
B=\frac{k \bar{p}}{M}, \quad j=2 g \beta^{-1} \sin \beta T / 2, \quad j^{\prime}=\partial j / \partial \beta,
$$

so that $j^{2}$ is the line shape of spontaneous emission and $\beta$ the detuning. The quantity $\delta$ is defined in Ref. 32 and turns out to be negligible under all circumstances. We shall in the following discuss Eqs. $(5.11-12)$.

Gain: The second term of the last line of Eq. (5.11) is the standard small-signal gain (cp. Eq. (3.17)) multiplied by the number of electrons. Thus there are no $0\left(\mathrm{~N}^{2}{ }^{2}\right)$ contributions to the gain for stimulated emission. This justifies the one-particle approach as far as gain is concerned.

Spontaneous emission: The first term in Eq. (5.11) is the usual spontaneous emission term, again multiplied by the number of electrons. The second (for $\mathrm{N}=0$ ) is a very small quantum correction. The third term which is absent for $N_{e}=1$, is again classical and not necessarily small. It can be referred to as amplified spontaneous emission. Initial spontaneous radiation which is proportional to $\mathrm{N}_{e}$ is afterwards amplified by one of the other electrons if gain is positive or attenuated otherwise, so that the total effect is proportional to $\mathrm{N}_{\mathrm{e}}\left(\mathrm{N}_{\mathrm{e}}-\mathrm{l}\right)$. As a consequence the observed spectrum of spontaneous emission deviates slightly from the commonly assumed $(\operatorname{sinx} / x)^{2}$ shape, as depicted in Fig. 4 for the parameters of the 1977 Stanford experiment $\left(\mathrm{J}=2.6 \mathrm{~A},(0.1 \mathrm{~cm})^{2}\right.$ electron beam area). Fig. 4

\section{FIGURE 4}

Spectrum of spontaneous emission versus detuning for the parameters of the Stanford 1977 experiment; dashed: usual $(\sin x / x)^{2}$ spectrum, solid line corrected for amplified spontaneous emission. The spectrum becomes negative for some values of $\beta \mathrm{T} / 2$ outside of the figure. This indicates that the firstorder expansion is no longer justified. Inclusion of higher orders would restore the spectrum to positive values.

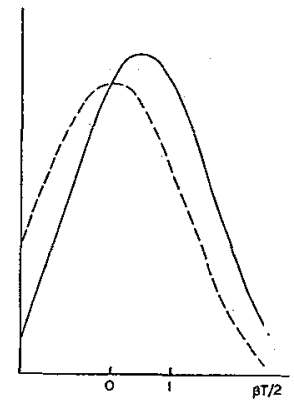


shows that the maximum of spontaneous emission is shifted towards the positive gain side by an amount which is proportional to the current density. Rewritten in the lab frame the shift in wavelength is

$$
\Delta \lambda=\pi /\left(10 \gamma^{3}\right)\left(e a_{w} / \mathrm{mc}^{2}\right)^{2} \mathrm{r}_{0} \lambda \mathrm{L}^{2}\left(\mathrm{~N}_{\mathrm{e}} / \mathrm{V}\right)
$$

with ro the classical electron radius. For the 1977 stanford parameters we obtain $\Delta \lambda=0.004 \mu$. Due to uncertainties in the electron energy and the wiggler field strength the position of the maximum of spontaneous emission cannot be measured with sufficient accuracy to detect this shift. However, Eq. (5.11) shows that gain is still given by the derivative of the spontaneous 1 ine shape for $\mathrm{N}_{e}=I$. Hence $\Delta \lambda$ should show up as a shift between the center of spontaneous emission and the position of zero gain.

Photon statistics of spontaneous emission: If the quantity $(\mathrm{N}=0)$

$$
\begin{aligned}
\Delta & =\Delta\left(N^{2}\right)-\Delta N=N_{e}\left(N_{e}-1\right) j^{4}-\frac{2 \hbar k^{2}}{M} N_{e}{ }^{2} j^{3} j \\
& + \text { terms of order } j^{6}
\end{aligned}
$$

is $>0,<0$, or $=0$, the photon statistics are super-poissonian, sub-Poissonian or coherent, respectively. In general, this corresponds to the emitted photons being bunched, anti-bunched, or coherent. For $\mathrm{N}_{e}=1, \Delta \mathrm{can}$ have either sign. Hence we have photon-antibunching for negative and photon-bunching for positive gain. (Note that gain is positive for $j^{\prime}<0$ in view of Eq. (5.11)). This is immediately obvious from physical arguments, cf. Fig. 5. In any event, the effect is extremely small. It would only become sensible for extremely long wigglers such that the spontaneous emission line shape is extremely narrow. Hence for $\mathrm{N}_{e}=1$ the photon statistics is very close to poissonian.

\section{FIGURE 5}

Spectrum of spontaneous emission versus detuning. For $\bar{p}<0$ the emission of one photon reduces the probability for the emission of another one, i.e. photon-antibunching occurs. The magnitude of $h_{k}$ is greatly exag gerated.

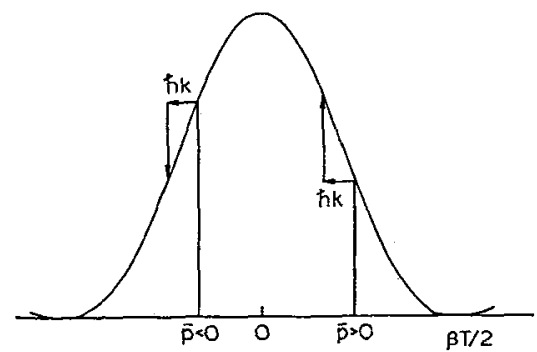

For $\mathrm{N}_{e}>1$ the first contribution to $\Delta$ is always dominant, indicating that spontaneous emission from several electrons is incoherent. However, one should note that the leading contribution from amplified spontaneous emission has dropped from Eq. (5.14). Hence this cooperative effect does not add additional incoherence, which gives a vague hint that finally the photon statistics of a free-electron laser might evolve towards a Poissonian distribution.

The noise over signal ratio for spontaneous emission is $(N=0)$

$$
\frac{\sqrt{(h \omega)^{2} \Delta\left(N^{2}\right)}}{\hbar \omega \Delta N} \cong\left[\left(1+\left(N_{e}-1\right) j^{2}\right) / N_{e} j^{2}\right]^{\frac{1}{2}}
$$

where we have, for an order of magnitude estimate, neglected the contributions from amplified spontaneous emission. In view of Eq. 
(5.4) and (5.13) we have $j^{2} w^{-1}$. Hence the first term in Eq. (5.15) is of quantum mechanical origin and can be attributed to the vacuum fluctuations of the electromagnetic field, while the second one is classical and is due to the discreteness of the electron current. In the lab frame we have

$$
N_{e} j^{2}=\frac{1}{2 \gamma^{2}} \frac{e^{2}}{\hbar c}\left(\frac{e a}{m c^{2}}\right)^{2} L^{2} \lambda\left(\frac{\sin \Delta \omega T / 2}{\Delta \omega T / 2}\right)^{2} \frac{N_{e}}{V}
$$

For $N_{e}=1$ the second term in Eq. (5.15) is absent and the noise is exclusively due to vacuum fluctuations. However, for the Stanford FEL

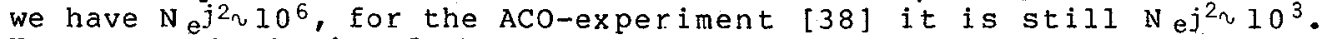
Hence on the basis of the fully quantized model outlined thus far one would conclude that for the existing high current FELs quantum mechanical contributions to the noise in spontaneous emission are negligible with respect to the classical ones.

Squeezing: The existence of squeezed states is a genuine quantum mechanical property of the electromagnetic field, which is outside of the scope of any classical description. They are defined in terms of the Hermitean components of the annihilation and creation operators a and $a^{\dagger}$

$$
a=a_{1}+a_{2}, \quad a_{i}=a_{i}{ }^{\dagger}(i=1,2),
$$

which satisfy the uncertainty relation

$$
\left(<\Delta a_{1}{ }^{2}><\Delta a_{2}{ }^{2}>\right)^{\frac{1}{2}} \geqslant \frac{1}{4} .
$$

A state is called squeezed if

$$
<\Delta a_{i}{ }^{2}><\frac{1}{4}
$$

for $i=1$ or 2. Experimental realization of squeezed states could have various interesting applications. As an example, we mention the possible improvement of the sensitivity of an interferometer with a view to the detection of gravitational waves (for a review, see [39]).

It has been shown that spontaneous radiation from a FEL is in a squeezed state [40]. We here consider the case $\mathrm{N}_{e}=1$. Then by means of the same methods that led to Eqs. (5.11-12) the result

$$
\left\langle\Delta A_{1}, 2^{2}\right\rangle=\frac{1}{4} \mp \frac{\hbar k^{2}}{2 M} j j \cdot
$$

is obtained. Hence depending upon whether gain is positive or negative, the state is squeezed with respect to $A_{2}$ or $A_{1}$. Here we took the operator $A=a \exp (i k z)$ defined in Eq. $(5.6)$ instead of the pure electromagnetic field operator a. As we remarked above with respect to photon-antibunching, the wiggler must be very long in order to render the effect sensible. For $\mathrm{N}_{\mathrm{e}}>1$ squeezing survives to some extent [33]. However, there are various doubts [33] as to whether this rather formal result might have practical significance. This is particularly the case for $\mathrm{N}_{\mathrm{e}}>\mathbf{l}$.

The coherence properties of a small-signal FEL-amplifier for $\mathrm{N}_{e}=1$ have been investigated in a somewhat different manner than discussed above in a series of papers $[37,41]$. The common starting point is the Hamiltonian $\left(5.1-3\right.$ ) (with $N_{=1}$ ), however, the interaction picture is not used. At some later stage, the electron motion is assumed to be classical and to follow a definite classical trajectory. The photon statistics are then (starting from the vacuum) poissonian at all times 
with a time-dependent mean value depending on the chosen trajectory. The Hamiltonian with the electron being classical preserves coherent states. In contrast, according to the approach followed above, the photon statistics developing from vacuum is only poissonian inasmuch as gain is negligible, and only under the same condition does the Hamiltonian rotate coherent states into each other. It appears that the two lines of approach deal with different physical situations: in $[32,33]$ the electron is initially described by a plane wave which corresponds to an average over classical initial conditions, i.e. the electron beam is initially unbunched. On the other hand, in $[37,41]$ the specification of a definite trajectory describes an initially bunched electron beam. The latter model has been pursued beyond tire small regime both for $N_{e}=1$ [42] and for $N_{e}^{>1}$ [43]. In these two papers the coherence properties and fluctuations have not been evaluated yet.

\section{ACKNOWLEDGEMENTS:}

We enjoyed discussions with G. T. Moore. This work was supported in part by the office of Naval Research. One of us (W.B.) acknowledges a Feodor-Lynen grant from the Alexander-von-Humboldt foundation.

\section{REFERENCES}

1. J. M. J. Madey, J. Appl. Phys. 42 (1971) 1906; J. M. J. Madey, H. A. Schwettman, and W. M. Fairbank, IEEE Trans. Nucl. Sci. 20 (1973) 980; W. B. Colson, Phys. Lett. 59A (1976) 187.

2. R. Pantell, G. Soucini and H. E. Puthoff, IEEE J. Quantum Electron. QE-4 (1968) 905; V. P. Sukhatme and P. W. Wolff, J. Appl. Phys. 44 (1973) 2331.

3. F. A. Hopf, P. Meystre, G. T. Moore and M. O. Scully in: Novel Sources of Coherent Radiation, Physics of Quantum Elecronics, Vol. 5 (Addison-Wesley, Reading, Mass., 1978), p. 41; N. M. Kroll, ibid., p. 115; W. B. Colson, ibid, p. 157.

4. See, e.g., M. Sargent, M. O. Scully and W. E. Lamb, Laser Physics (Addison-Wesley, Reading, Mass. 1974).

5. See, e.g., S. Schweber, Relativistic quantum Field Theory (Harper and Row, New York, 1962).

6. W. Becker and H. Mitter, Zs. Phys. B35 (1979) 399.

7. See, e.g. J. Bjorken and S. D. Drell, Relativistic Quantum Mechanics (McGraw-Hill, New York, 1964).

8. H. Motz, Phys. Lett. 71A (1979) 41.

9. R. J. Glauber, Phys. Rev. 84 (1951) 395.

10. R. T. Deck and P. G. Gill, Phys. Rev. A26 (1982) 423)

11. J. Walsh, 1981 Snowbird Conference on Quantum Electronics, unpublished.

12. D. F. Zaretskii, E. A. Nersesov and M. V. Fedorov, Zh. Eksp. Teor. Fiz. 80 (1981) 999 [Sov. Phys. JETP 53 (1981) 508].

13. W. A.McMuli in and R. C. Davidson, Phys. Rev. A25 (1982) 3130.

14. G. Bekefi, Radiation Processes in Plasmas (Wiley, New York, $1966)$.

15. W. Becker, Opt. Commun. 33 (1980) 69.

16. W. Becker, Zs. Phys. B42 (1981) 87.

17. W. Becker, Physics of Quantum Electronics, Vo1.9 (Add i son-Wesley, Reading, Mass., 1982), p. 985.

18. A. Bambini and S. Stenholm, Opt. Commun. 30 (1979) 391.

19. W. Becker, Opt. Commun. 36 (1981) 64.

20. S. T. Stenholm and A. Bambini, IEEE J. Quantum Electronics $Q E-17$ (1981) 1363.

21. J. M. J. Madey, Nuovo Cimento 50B (1979) 64; N. M. Kroll, Physics of Quantum Electronics Vol . 8 (1982) p. 315.

22. R. Bonifacio, P. Meystre, G. T. Moore and M. O. Scully, Phys. 
Rev. A21 (1980) 2009.

23. W. Becker and J. K. McIver, Phys. Rev. A25 (1982) 956.

24. J. Soln, J. Appl. Phys. 52 (1981) 6882.

25. J. K. MCIver, M. V. Fedorov, Zh. Eksp. Teor. Fiz. 76 (1979) 1996

[Sov. Phys. JETP 49 (1979) 1012].

26. M. V. Fedorov and J. K. McIver, Phys. Lett. 72A (1979) 83.

27. W. Becker, Zs. Phys. B38 (1980) 287.

28. M. V. Fedorov, Prog. Quant. Electr. 7 (1981) 73.

29. J. N. Eckstein, J. M. J. Madey, K. Robinson. T. I. Smith, S. Benson, D. Deacon, R. Taber and A. Gaupp in: Free-Electron Generators of Coherent Radiation, Physics of Quantum Electronics, Vol. 8 (Addison-Wesley, Reading, Mass., 1982) p. 49.

30. J. K. McIver and M. V. Fedorov, Kvant. Elektron. 7 (1980) 309 [Sov. J. Quant. Electron. 10 (1980) 1977]; M. V. Fedorov and J. K. McIver, Optica Acta 26 (1979) 1121.

31. W. H. Louisell, J. F. Lam, D. A. Copeland and W. B. Colson, Phys. Rev. Al9 (1979) 288 .

32. W. Becker and M. S. Zubairy, Phys. Rev. A25 (1982) 2200.

33. W. Becker and J. K. McIver, Phys. Rev. A (in print).

34. A. Bambini and A. Renieri, Lett. Nuovo Cimento 31 (1978) 399; Opt. Commun. 29 (1978) 24; A. Bambini, A.Renieri and S. Stenholm, Phys. Rev. Al9 (1979) 2013.

35. A. Bambini and S. Stenholm, Optica Acta 27 (1980) 201.

36. G. Dattoli, Lett. Nuovo Cimento 27 (1980) 247.

37. R. Bonifacio, Opt. Commun. 32 (1980) 440.

38. C. Bazin, M. Billardon, D. A. G. Deacon, P. Elleaume, Y. Farge, J. M. J. Madey, J. M. Ortega, Y. Petroff, K. E. Robinson, M. Velghe, Physics of Quantum Electronics, Vol. 8 (Addison-Wesley, Reading, Mass. 1982), p. 89 .

39. C. M. Caves, K. S. Thorne, P. W. P. Drever, V. D. Sandberg, and M. Zimmermann, Rev. Mod. Phys. 52 (1980) 341.

40. W. Becker, M. O. Scully and M. S. Zubairy, Phys. Rev. Lett 48 (1982) 475.

41. G. Dattoli, A. Renieri, F. Romanelli, and R. Bonifacio, Opt. Commun. 34 (1980) 240; G. Dattoli, A. Renieri, and F. Romanelli, Opt. Commun. 35 (1980) 245; G. Dattoli and A. Renieri, Opt. Commun. 39 (1981) 328 .

42. R. Bonifacio, F. Casagrande and L. A. Lugiato, Opt. Commun. 36 (1981) 159.

43. R. Bonifacio, F. Casagrande and G. Casati, Opt. Commun. 40 (1982) 219 . 